\title{
Data Visualization in Evaluating Service Delivery
}

\author{
Randy Basham \\ University of Texas at Arlington, Texas, United States
}

\begin{abstract}
The delivery of human services has become highly complex and is increasingly reliant on the capacity of experienced local service providers to evaluate complex datasets so as to provide adequate decision-support for critical and costly service delivery needs. Traditionally, localized human service agencies which are of relatively small size have limited resources and must control administrative expenditures to provide human services.
\end{abstract}

Keywords: performance evaluation, computing, data visualization, group evaluation, human service delivery

\section{Introduction}

Human service and health service organizations are in critical need of developing best practice, technical and evaluative tools, that keep pace with rising social expectations for evaluation of health and service delivery methods. The needs and numbers of health service recipients are changing dramatically in recent decades. Contemporary life for the most needy of our world's residents has become more urgent and critical with respect to responding to seemingly pandemic suffering, oppression, and inequities related to the disparities of inconsistently distributed goods and services. Geopolitical events, and economic and natural catastrophes have created reservoirs of expectant and well-deserving people who are increasingly relying on social workers and other human service providers, to assist them in individual and collective humanitarian collective recovery efforts (Basham \& Diaz, 2005). Though data visualization has been well received and integrated into medical service care, computing science, and engineering, and meteorology, it has received less attention in the delivery of social service and the exploration of data or evaluation of date to determine service delivery effectiveness. Data visualization can be utilized to determine whether service recipients are receiving equivalent benefit or outcomes through multilevel graphical evaluation methods using available desktop computing technology (Jelen, 2015).

Small or rural agency health and human services providers of service delivery methods and interventions often remain to some extent mired in the service delivery and service evaluation technologies and traditions of the middle of the last century. These service providers are challenged in the effective delivery of services by increasing service need and static unchanging delivery and evaluative methods. Transforming current and emerging technologies to serve the needs of the many and to provide meaningful decision-support methods for service providers, knowledge developers, and educators in meeting these needs has never been more vital (Patterson \& Basham, 2003). There is sufficient service need for smaller human service delivery organizations to move reduce dependence on proprietary and expensive data analysis packages and to utilize more available spreadsheet applications which are affordable and represent both emerging and substantially improved tools for educating health care practitioners and as tools for evaluating practice and service delivery.

Randy Basham, Ph.D., Associate Professor, School of Social Work, University of Texas at Arlington, Texas, United States. 
This paper addresses emerging best practices in the incorporation of widely available desktop computing technologies to provide data visualization or graphical representation methods to evaluate services to small groups of health and social service recipients, within less developed service delivery agencies. This session will also present discuss and demonstrate methods being developed at the University of Texas at Arlington, School of Social Work to utilize data visualization methods to evaluate small group service delivery efforts and to utilize spreadsheet methods of analyzing data in a widely available desktop computing application (Microsoft Excel) as an alternative to expensive specialized data analysis packages. The presentation will demonstrate graphical representation methods of visually evaluating process and outcome simultaneously over time, as well as evaluating multiple units of analysis or levels of service graphically. Furthermore, multimedia modules are demonstrated that rapidly teach complex computing procedures using spreadsheets to analyze common human service data problems (Basham, 2002).

Digital or e-learning strategies, incorporating widely available graphical representation tools to evaluate service, combined with streaming audio and video modules to teach, or transfer complex evaluative data analytic technologies and knowledge show promise of greatly improving service delivery and service related education relative to evaluation. Social service delivery need are rapidly changing as service providers become accountable primarily to service constituencies competing for a greater share of service delivery and goods, regulatory boards, payers of funded services, and face increasing legal challenges to service equity and effectiveness. Therefore, this paper addresses two recent integrations of best practices in desktop computing technologies to both render graphical representation for data to assist practice and administrative decision support and evaluative service to smaller service agencies using spreadsheet computing.

\section{Method 1: Data Visualization in the Evaluation of Service Delivery}

A study was undertaken to determine the utility of widely available desktop computing technology to provide graphical representations of data sufficient to provide rich contextual and multilevel evaluative information to educators on the development of cohesion and dimensions of group process at the level of individuals and groups simultaneously. Measures of comparative group leadership across multiple group service offerings using the spreadsheet tolls and graphing capacities of the Microsoft Excel application were also evaluated. The project entitled "Data Visualization: Graphical Representation in the Evaluation of Experiential Group Therapy Education Outcomes” (Basham, 2002; Patterson \& Basham, 2002) is summarized.

\section{Purpose}

The purpose of the study was to quantitatively and graphically evaluate the usefulness of data visualization methods to represent group level change over time. Group level educational outcomes were analyzed by using conventional data analytic methods for time series data and through use of widely available computing technology, to graphically represent change over time (see Figures 1 and 2). Figure 1 depicts the capacity of the spreadsheet package to render data visualization that captures group turbulence and cohesion across time using a moving standard deviation via a standard deviation enhanced line graph (SDELG), computed, and graphed across multiple periods of observation.

\section{Methods}

This paper reports findings from group level time series data for 16 experiential group therapy education groups that included 249 subjects and were evaluated using quantitative and visual analytic procedures. 
Quantitative analytic procedures included: descriptive and inferential analysis to demonstrate limitations to statistical conclusion validity when working with non-probability samples, psychometric reliability, and validity analysis of the survey instrument to include an exploratory principal axis factor analysis. Visual analytic procedures included traditional methods of visual evaluation of single subject information, as well as, less common graphical representation methods.

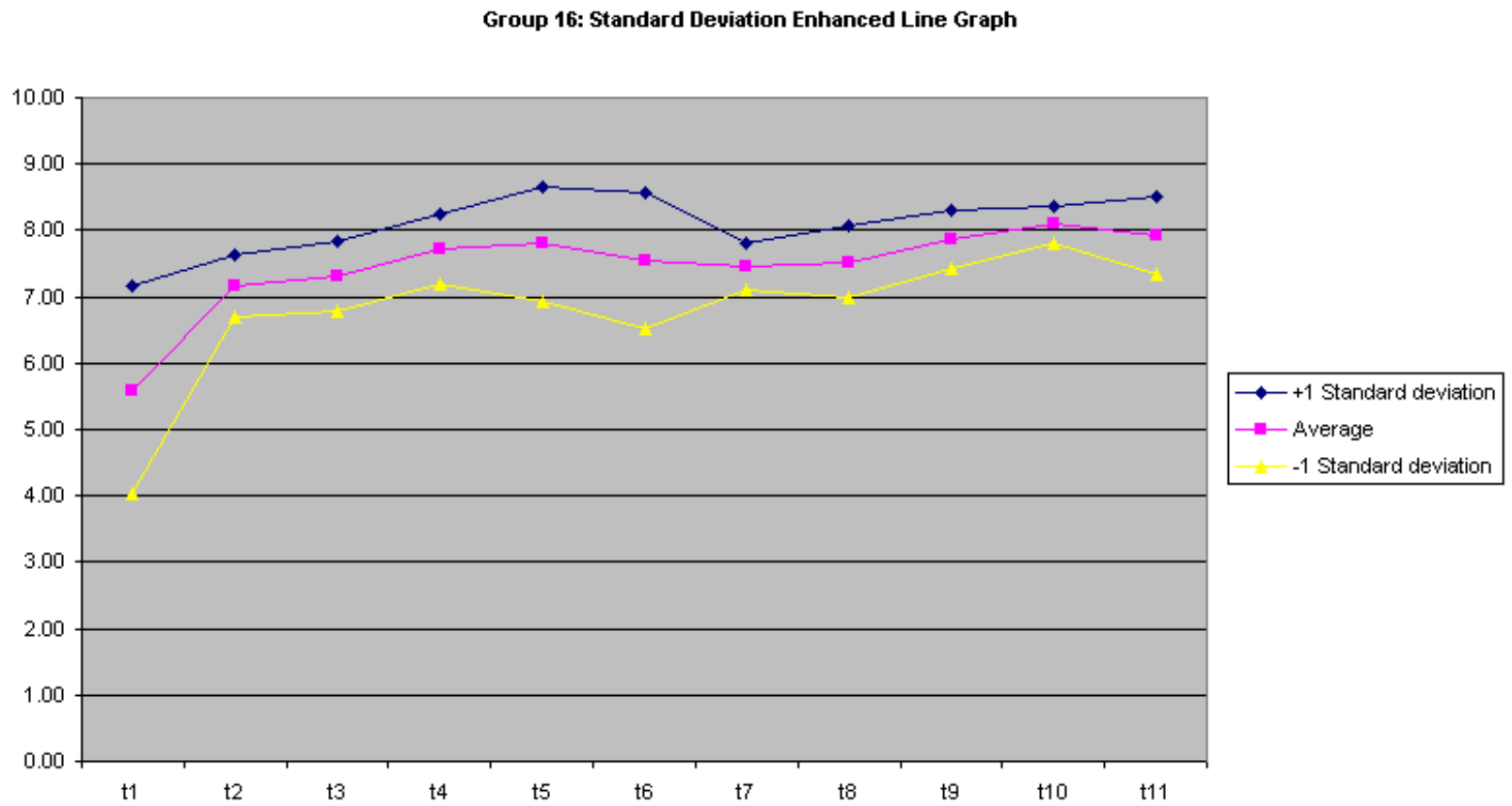

Figure 1. Group 16 (Excel)-SDELG: Concordance and variation of group satisfaction across weeks (Traditional single subject line graph of group score average per time of observation is not shown).

\section{Results}

The resultant graphical representation demonstrates the difficulty of using statistical analysis procedures with non-probability samples. Psychometric analysis of the survey instrument though suggests a highly reliable group level evaluative instrument (coefficient alpha $r=0.90$ ). Data visualization methods simultaneously illustrated levels of group participant concordance and variability over time. Graphics were also generated that demonstrate the proportional contribution of multiple variables to group outcomes over time and the simultaneous display of process and outcome indicators over time. Furthermore, methods presented demonstrated the capacity to represent multiple units of analysis over time, and multiple groups of non-equivalent duration, for comparison across time. Figure 2 demonstrates comparative leadership scores averages plotted on a three dimensional surface plot for group leaders conducting educational training groups across time with groups of differing numbers of total group meeting sessions.

\section{Implications for Practice}

Findings indicate that data visualization methods are highly useful in evaluating and understanding the complexities of multilevel data common to the delivery of group services. Graphical understanding can be improved further by the inclusion of relevant data analytic procedures to identify statistical conclusion validity issues. 


\section{Multiple Groups: Surface Plot-Group Mean Satisfaction by Variable Length Groups and by Leaders Across Weeks}

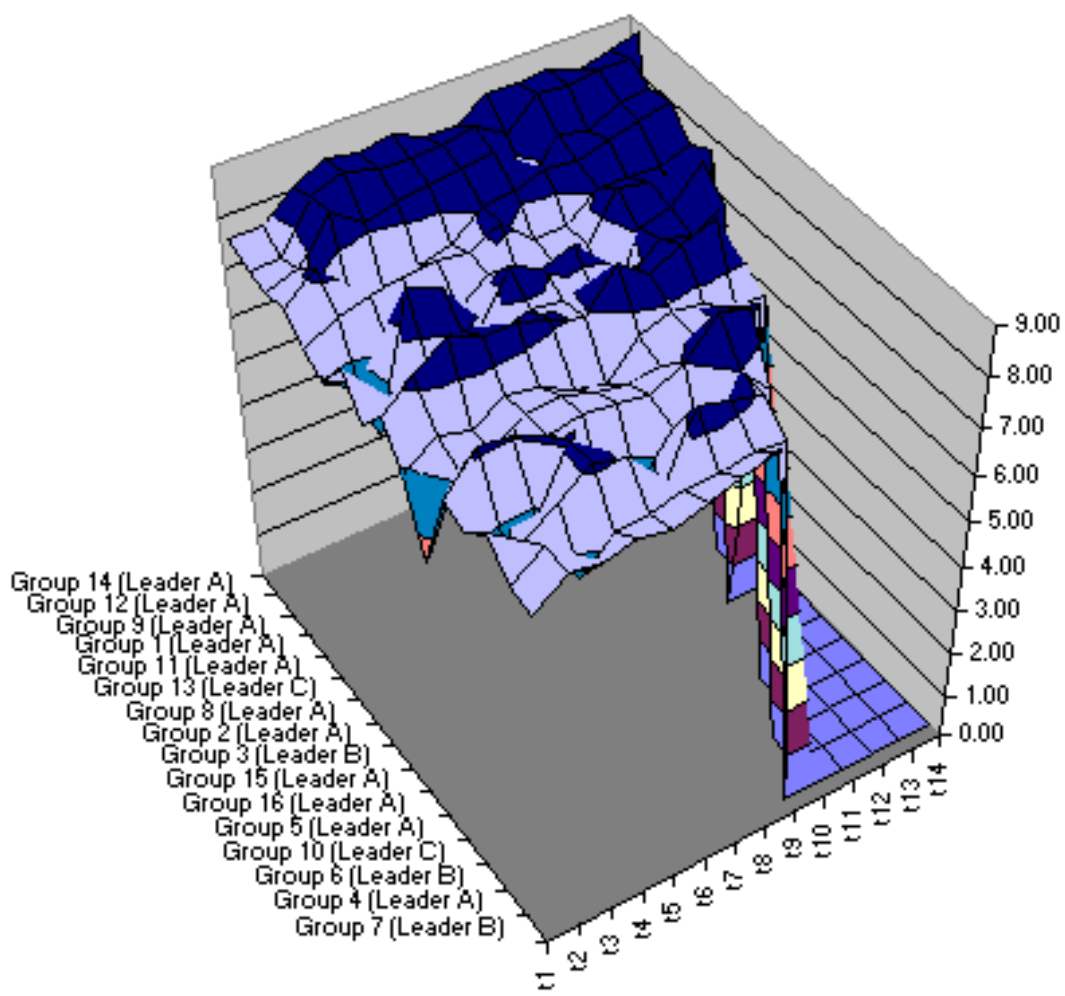

Figure 2. Multiple Groups (Excel)-Three-dimensional surface plot: Group satisfaction by group leaders.

\section{Method 2: Data Visualization in Educating Service Providers in Data Analysis}

Microsoft Excel spreadsheet application also have a number of advanced statistical function, the capacity for pivot table reports and charting, and advanced add in statistical computing modules already contained within the program. However, unlike commonly used advanced statistical computing packages, these spreadsheet statistical computing options are not conveniently menu driven. However, the graphical and data visualization options are generally and greatly enhanced, as compared to common social science oriented academic statistical computing packages. Using screen capture technology and streaming audio and video modules the e-learning oriented educator can provide screen capture instruction with audio direction so that the student can quickly master essential statistical computing skills on an application that is generally available throughout most smaller scale human service delivery organizations. The integrated graphing package can also provide highly useful data exploration, trend analysis, and visual confirmation to provide enhanced decision support for vital human service delivery and intervention effectiveness evaluation.

Service delivery knowledge development is partially constrained by the fact that service providers are not well-prepared to conduct data analysis with the most commonly available desktop technology applications, such as the spreadsheet. In the education of service providers, statistical software packages, such as Statistic Package for Social Science (SPSS) or Statistical Analysis System (SAS), are commonly used to teach data analysis and statistical procedures (Dzemyda, 2005). Statistical software packages commonly used for 
advanced service practitioner education are both expensive and commonly unavailable in most small-scale service delivery settings. Educators can readily integrate spreadsheet data analysis into research and statistics courses with the likelihood of greater utility for small agency service providers and greater post-graduate generalization to the service delivery environment (Patterson \& Basham, 2006a).

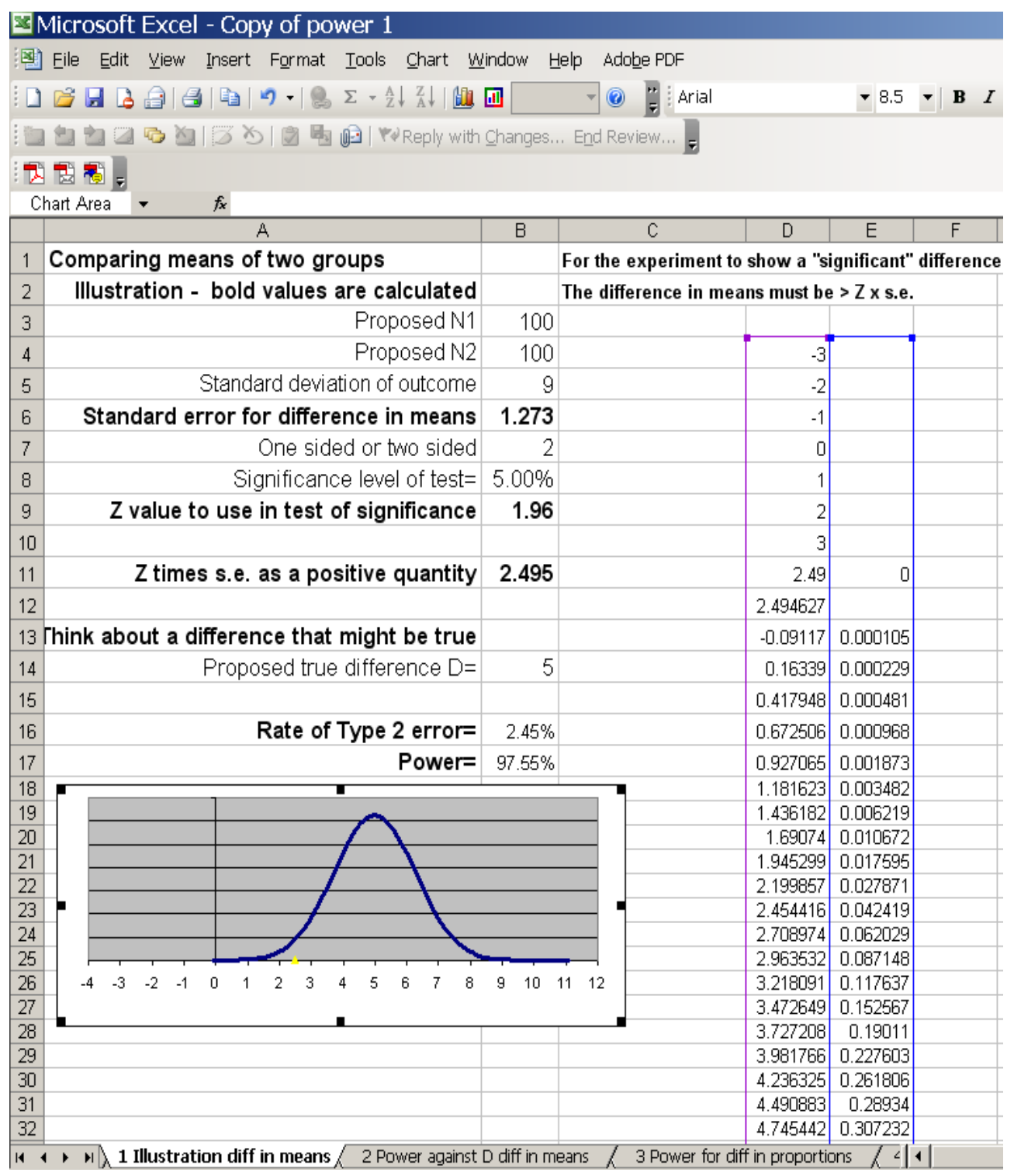

Figure 3. A screen capture of a statistical procedure along with a graphical representation.

Instructors can quickly master the complexities of statistical and data analysis tools available in spreadsheet software. These available tools include spreadsheets in data collection, sampling, data cleaning, frequency distributions, histograms, descriptive statistics, inferential statistics, bivariate statistics, pivot tables (cross-tabs), single system designs, correlation, and graphical representation of results (Basham, 2001). Additional support tools available for educators and e-learning students include sorting procedures, pivot tables, functions, statistical analysis tools, auto filters, and chart wizard (Broman \& Woo, 2018). By organizing learning module a portable or web based delivery media, the instructor can make audio and video learning 
modules available for rapid replication and learning, as well as providing a means for skill generalization independent form the classroom setting. In Figure 3, a comparison of means of two groups using a spreadsheet has been screen captured, but may also be captured by screen recording software to demonstrate each cursor move of the instructor and each dialog box decision in setting up the problem. The screen animated capture can also be dubbed, with voice over technology providing a complete learning module (Patterson \& Basham, 2006b).

\section{Discussion}

Results from the first method or study indicated that data visualization methods are highly useful in evaluating and understanding the complexities of multilevel data common to the delivery of group services. This is especially true when the survey or measurement instrument of the group attribute of interest is a highly reliable measurement or assessment instrument. Graphical understanding can be improved further by the inclusion of relevant data analytic procedures to identify statistical conclusion validity issues, especially when non-random samples are not available for evaluation (Wagner \& Keisler, 2006). Human service workers and those providing group services to large populations, such as human service programs are in need of developing practice accountability strategies to evaluate group services that include data visualization methodology. These graphical innovations provide additional data exploration capacity and contribute greatly to understanding of data when the graphic selected is consistent with the type of data to be evaluated and adheres to graphing conventions, such as reporting test statistics in context with the presentation of the graphic. This exploratory study does not resolve, however, those issues of best utilization of graphic type per problems of interest that will require additional field level investigation. However, the question of acceptance by various practitioners and ease of use must be deferred here pending further study.

The second method of educating service providers through e-learning modules to evaluate services using statistical software and graphing technologies found in a widely available desktop computing technology also offer greater generalization capacity into the service delivery environment. Spreadsheet technology may be a cost effective and quickly transferable method of providing skill sets needed for consistent decision support in the provision of human services. Digital multimedia modules containing animated screen captures and voice instruction may be taken to the work environment and reviewed and then implemented over short period. Service evaluators can select only those modules or data analytic and graphing capacities relevant to a specific problem in the field environment.

\section{Conclusion}

Utilizing modern data analytic software that is widely available (MS Excel), behavioral scientists and other service delivery professionals can evaluate multiple units of analysis across multiple groups, multiple dimensions of measurement, and across time while limiting possible ecological fallacy interpretations. Complex performance data can be rendered through advanced graphical representation tools that are color enhanced, rendered in three dimensions, and with rotatable axes that permit improved clinical and statistical conclusion validity, even for replicated observations, through visual inspection and analysis. Service providers and behavioral health specialists can readily determine differences between individual comparative performance and group level comparative performance across time. Examples of fully rotatable three-dimensional graphical representations of performance and be rendered offering improved understanding 
of complex human performance issues. Methods of developing and utilizing these tools and be developed into learning modules for training purposes using also widely available voice over and screen capture tools.

\section{References}

Basham, R. (2002, December). Data visualization: Graphical representation in the evaluation of experiential group therapy education outcomes. Dissertation Abstracts International Number: 3086821, Vol. 64-02A.

Basham, R. E. (2001). Multivariate regression, ordinary least squares. In D. A. Patterson (Ed.), Using spreadsheets for data collection, statistical analysis and graphical representation [CD-ROM]. Retrieved from http://web.utk.edu/ dap/Random/Order/Start

Basham, R. E., \& Diaz, H. L. (2005). Cómo visualizar las necesidades: Uso de técnicas de visualización como instrumentos de apoyo en la formulación de la política social (Visualizing need: Using data visualization as a decision-support tool in the formulation of social policy). In de M. R., Ferreira, \& R. E. L., Estrada (Eds.), Tópicos selectos en politicas de bienestar social, Tomos I y II (Selected topics in social welfare policy) (Volumes I and II, pp. 42-50). Trillas Publishing, NL, Monterrey, Mexico.

Broman, K. W., \& Woo, K. H. (2018). Data organization in spreadsheets. American Statistician, 72(1), 2-10. doi: $10.1080 / 00031305.2017 .1375989$

Dzemyda, G. (2005). Multidimensional data visualization in the statistical analysis of curricula. Computational Statistics \& Data Analysis, 49(1), 265-281.

Jelen, B. (2015). Excel visualizing positive and negative changes. Strategic Finance, 97(8), 82-83.

Patterson, D. A., \& Basham, R. E. (2002). Data visualization procedures in the analysis of group treatment outcomes across units of analysis. Small Group Research, 33(2), 209-233.

Patterson, D. A., \& Basham, R. E. (2003). Visualizing change: Spreadsheets and graphical representation across domains in human service practice. Journal of Technology in Human Services, 21(4), 1-16.

Patterson, D. A., \& Basham, R. E. (2006a). Data analysis with spreadsheets. Boston, Massachusetts: Allyn \& Bacon Publishers. ISBN 0-205-40751-X

Patterson, D. A., \& Basham, R. E. (2006b). Data analysis with spreadsheets: Multimedia modules (CD-ROM). Boston, Massachusetts: Allyn \& Bacon Publishers. ISBN 0-205-40751-X

Wagner, J. M., \& Keisler, J. (2006). Enhance your own research productivity using spreadsheets. In M. P. Johnson, B. Norman, \& N. Secomandi (Eds.), Models, methods, and applications for innovative decision making: INFORMS tutorials in operations research (pp. 148-162). Catonsville, MD: INFORMS. Retrieved from https://doi.org/10.1287/educ.1063.0028 